\title{
"Evaluación del liderazgo del equipo directivo y su relación con el desempeño de los docentes de la I.E. 0069 Machu Picchu Red 09- UGEL 05 San Juan de Lurigancho -2015"
}

\author{
"Evaluation of the leadership of the management team and its \\ relation with the performance of the teachers of the I.E. 0069 \\ Machu Picchu Red 09- UGEL 05 San Juan de Lurigancho -2015 "
}

\author{
Sofía Gamarra Mendoza \\ Escuela Profesional de Educación / Universidad Nacional Federico Villarreal
}

\begin{abstract}
RESUMEN
El presente trabajo de investigación es el fruto del gran esfuerzo y privaciones familiares, para investigar acerca de la relación del liderazgo del equipo directivo y como este influye en el desempeño docente de su institución, con el firme propósito de mejorar la calidad de la educación en nuestro país.

En estos tiempos, el liderazgo propuesto por el del maestro líder se ha convertido en un elemento básico de cualquier intento de modernización educativa y con ello el mejoramiento del desempeño docente, por lo tanto, es impostergable la necesidad de investigar y publicar los resultados obtenidos en esta modesta investigación para ayudar a quienes tienen la responsabilidad de dirigir instituciones educativas a nivel nacional.

En tal sentido, se analiza el liderazgo empleado por los directivos para poner en acción a su equipo para la obtención de logros y metas que tiene una relación directa con el desempeño docente que muestren los maestros bajo su dirección y, para lograrlo es indispensable que estas metas sean fijadas de común acuerdo, sólo así es posible despertar el interés y la motivación para trabajar juntos hasta conseguir lo propuesto.
\end{abstract}

Palabras claves: Gestión pedagógica, calidad del servicio educativo.

\begin{abstract}
This research is the result of great effort and family deprivation, to investigate the relationship management leadership and how this influences the teaching performance of their institution, with the firm intention of improving the quality of education in our country.

In these times, leadership proposed by the lead teacher has become a staple of any attempt to educational modernization and thereby improving teacher performance, therefore it is urgent the need to investigate and publish the results of this modest research to help those who have the responsibility of leading educational institutions nationwide.

In this sense, leadership employee is analyzed by managers to put into action his team to obtain achievements and goals that have a direct relationship to teacher performance that show teachers under his direction and to achieve this it is essential that these goals to be set by mutual agreement, only then can arouse interest and motivation to work together to achieve proposed.

I am pleased then, put in your hands this small contribution, the result of the dedication and concern for the generations that come behind, hoping a boon to them, apologizing for the shortcomings they might encounter
\end{abstract}

Keywords: Educational Management - Quality Educational Service. 


\section{Introducción}

El desempeño docente es fundamental para crear una influencia positiva en el aprendizaje significativo de los alumnos. Es, por tal motivo, necesario analizar los factores que benefician o perjudican el desempeño adecuado y que puedan ser beneficiosos a nuestros maestros.

De igual forma, los estilos de liderazgo son componentes fundamentales en su desempeño, por cuanto proporcionan al docente una serie de estrategias y habilidades esenciales para su buena influencia y planificación académica. Es por ello que, el presente estudio determine el tipo de relación que existe entre ambas variables, y pretenda ser una guía para hacer énfasis en la formación y dirección de estos estilos de liderazgo, que redunde en beneficio de los estudiantes.

Padilla, Dante (2005), sostienes que las habilidades de liderazgo de dirección estratégica, cohesión, negociación y toma de decisiones, la respuesta más común es la de indeterminación. Es decir, los públicos estudiados no reconocen estas habilidades en ellos mismos" (pg. 47).

Trujillo, María (2004) Gestión Educativa, en Lima, menciona: "Cada institución educativa tiene su propia cultura, distinta de las demás, lo que le da su propia identidad. La cultura de una institución incluye los valores, creencias y comportamientos que se consolidan y comparten durante la vida educativa

Rincón, J. (1995) Relación entre estilo de liderazgo del director y desempeño de docentes del Valle de Chumbao de la provincia de Andahuaylas. De los resultados obtenidos los datos nos evidencian que existe un alto grado de correlación entre el estilo de liderazgo del director y el desempeño docente en las Instituciones Educativas del Valle de Chumbao de la provincia de Andahuaylas. Se puede deducir que el desempeño docente depende del estilo de liderazgo del director.

Flores, R. (2003) Estilos de liderazgo y su relación con el desempeño docente en el aula, según la percepción y evaluación de los alumnos del quinto grado de secundaria en los colegios estatales de áreas técnicas de la USE Nº6 ATEVITARTE. Al realizar esta investigación se llegó a la conclusión de que los docentes en su desempeño en el aula utilizan con mayor frecuencia y en forma predominante el estilo de liderazgo tolerancia a la libertad; en un nivel alto predomina el estilo de liderazgo consideración y en un nivel medio predomina el estilo de liderazgo Iniciación de estructura.

Rosales, Mariela (2005), en su trabajo "Calidad sin Liderazgo", analizó las conductas de liderazgo de siete directores de escuelas de clase media en la provincia de San Juan - Argentina. Población: directores y profesores. Diseño de investigación: Se realizó una investigación descriptiva utilizando técnicas de observación, entrevista, encuestas y cuestionarios de auto evaluación. En sus conclusiones expresa, "que los directores escolares presentan conductas más de administradores que de líderes".

Benvenuto, Jobita, Monge, Zully \& Zanini, Marcela (2005), en su investigación sobre "La Incidencia de las Relaciones Interpersonales en el desempeño escolar “, Venezuela. Metodología: Es un diseño descriptivo. Concluyen, "Nuestra afirmación el rol de cada docente influye en la comunicación y en el desempeño escolar del equipo directivodocente...".

¿En qué medida se correlaciona la Capacidad de Liderazgo del Equipo Directivo y el Desempeño Docente de la I.E. 0069 Machu Picchu RED 09UGEL 05 San Juan de Lurigancho?

Asimismo, se presentan las conclusiones en torno al trabajo de investigación y se aportan algunas sugerencias para los docentes.

Se espera que el presente trabajo aporte algunas ideas para el docente en el aula como una herramienta eficaz que facilite, estimule $y$ garantice un aprendizaje significativo para los niños. El cual esperamos sea de mucho provecho, pidiéndoles las disculpas por los errores que se pudieran encontrar.

\section{Material y métodos}

\section{Objeto del estudio}

El objeto de estudio estuvo constituido por Equipo Directivo y Docentes de la I.E. 0069 Machu Picchu RED 09- UGEL 05 San Juan de Lurigancho 2015.

\section{Población}

La población estuvo constituida 70 docentes de la I.E. 0069 Machu Picchu RED 09- UGEL 05 San Juan de Lurigancho.

\section{Muestra}

Del total de la población se trabaja con 60 docentes de la I.E. 0069 Machu Picchu RED 09UGEL 05 San Juan de Lurigancho.

\section{Instrumentos de recolección de datos}

- Observación Documental. Permite determinar el problema y elaborar aspectos teóricos a través de fichas bibliográficas.

- Ficha de observación. Recojo de información a través de cuaderno de ocurrencias

- Encuestas. Recojo de información proveniente de docentes a través de pruebas estandarizadas 


\section{Métodos y Técnicas}

Método correlativo correlacional

Técnica

Observación

Diseño de estudio

El diseño de la investigación es de carácter descriptivo correlacional.

Procedimiento:

- Para el efecto de la recolección de datos se seleccionó como técnica la entrevista y como instrumento se utilizó el cuestionario, el cual fue aplicado en forma personal a cada docente por parte de la investigadora; considerándose a estos como medios efectivos para recolectar datos reales sobre el presente estudio siendo su objetivo el de obtener información.

- Posteriormente los datos se procesaron, analizaron y se discutieron

- Se redactó las conclusiones y sugerencias en base a la discusión de resultados

- Se comunicaron las conclusiones del trabajo de investigación.

- La validez del instrumento, se hizo mediante el juicio de expertos, participando profesionales que laboran en la docencia y del área de Investigación; cuyas opiniones y recomendaciones sirvieron para mejorar el instrumento. Los puntajes fueron sometidos a la prueba binomial, encontrándose que el error de significancia no supera los valores establecidos para $\mathrm{p}<0.05$ (anexo “B”), por lo que el instrumento es válido.

La confiabilidad del instrumento se determinó a través de la aplicación de una prueba piloto que se realizó, tomando a 30 docentes de muestra piloto con similares características a la del estudio pero que no pertenece a la población. Así se aplicaron formulas estadísticas tales como el coeficiente de alfa de Crombach para la confiabilidad de conocimientos, obteniéndose como resultado: el instrumento que se utilizó para medir el desempeño docente es el Alfa de Crombach $=0.96 \mathrm{y}$ liderazgo del equipo directivo el Alfa de Crombach $=0.98$ muy alto. Habiéndose encontrado resultado confiable y consistente a través del tiempo para todo el instrumento en general.

- Observándose que luego de su aplicación, los resultados son confiables.

- Estos resultados permitieron reestructurar y/o discriminar ítems, garantizando su consistencia y coherencia interna, así como reducir al máximo el margen de error.

\section{Resultados}

Para un mejor tratamiento estadístico de los datos, haremos uso del Software Informático SPSS V15 en Programa Excel, de Microsoft Office, Versión 2005.

Para efectos del tratamiento estadístico de la información se hará uso de los siguientes procedimientos:

Coeficiente de correlación de Pearson (Hernández Sampieri, R y otros. 1996, Metodología de la Investigación: Mc Graw Hill), (16):

Definición: Es una prueba estadística para analizar la relación entre dos variables medidas en un nivel por intervalos de razón.

$$
\begin{aligned}
& \text { Se simboliza }=\mathrm{R} \\
& \qquad R^{2}=\frac{\sum X Y-\sum X \sum Y}{\sqrt{\left(N \sum X^{2}-\left(\sum x\right)^{2}\right)\left(N \sum Y^{2}-\left(\sum Y\right)^{2}\right)}}
\end{aligned}
$$

El trabajo de investigación para ser probado se ha tenido que diseñar tres hipótesis de estudio, y como consecuencia de ello se ha diseñado dos modelos de encuesta, donde cada encuesta tratara de probar cada uno de las hipótesis, los cuales probaran la hipótesis principal: Existe una correlación significativa entre la capacidad de liderazgo del equipo directivo y el desempeño docente de la I.E. 0069 Machu Picchu RED 09UGEL 05 San Juan de Lurigancho. Las específicas que se plantean en el trabajo de investigación son las que se muestra a continuación:

\section{Hipótesis 1}

H1: Existe una correlación directa entre la comunicación del equipo directivo y el desempeño docente de la I.E. 0069 Machu Picchu RED 09UGEL 05 San Juan de Lurigancho.

Ho: No existe una correlación directa entre la comunicación del equipo directivo y el desempeño docente de la I.E. 0069 Machu Picchu RED 09UGEL 05 San Juan de Lurigancho. 


\section{Hipótesis 2}

H1: Existe una correlación importante entre la motivación del equipo directivo y el desempeño docente de la I.E. 0069 Machu Picchu RED 09UGEL 05 San Juan de Lurigancho Ho: No existe una correlación importante entre la motivación del equipo directivo y el desempeño docente de la I.E. 0069 Machu Picchu RED 09- UGEL 05 San Juan de Lurigancho.

\section{Hipótesis 3}

H1: Existe una correlación positiva entre el liderazgo del equipo directivo y el desempeño docente de la I.E. 0069 Machu Picchu RED 09UGEL 05 San Juan de Lurigancho.

Ho: No existe una correlación positiva entre el liderazgo del equipo directivo y el desempeño docente de la I.E. 0069 Machu Picchu RED 09UGEL 05 San Juan de Lurigancho.
Para probar estas hipótesis se han diseñado un cuestionario, que mide el desempeño docente y el liderazgo, tiene 38 preguntas que son politómicas ponderadas (Escala de Likert) Se ha encuestado a 60 docentes de la I.E. 0069 Machu Picchu RED 09- UGEL 05 San Juan de Lurigancho.

\section{Primera Prueba de Hipótesis}

\section{Hipótesis de Trabajo}

H1: Existe una correlación directa entre la comunicación del equipo directivo y el desempeño docente de la I.E. 0069 Machu Picchu RED 09UGEL 05 San Juan de Lurigancho.

Ho: No Existe una correlación directa entre la comunicación del equipo directivo y el desempeño docente de la I.E. 0069 Machu Picchu RED 09UGEL 05 San Juan de Lurigancho.

Formula del coeficiente de correlación de Pearson

$$
R=\frac{\sum X Y-\sum X \sum Y}{\sqrt{\left(N \sum X^{2}-\left(\sum x\right)^{2}\right)\left(N \sum Y^{2}-\left(\sum Y\right)^{2}\right)}}
$$

Tabla 1. Contraste entre La Dimensión Comunicación y Desempeño Docente

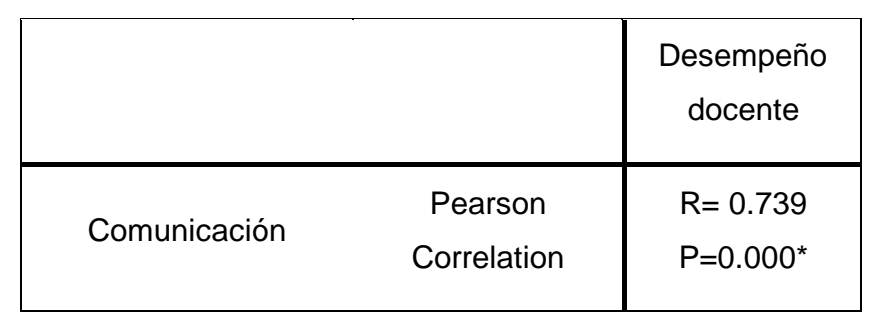

$* * \mathrm{P}<0.05$ significativo

Existe una correlación positiva (0.73) entre la comunicación del equipo directivo y el desempeño docente de la I.E. 0069 Machu Picchu RED 09- UGEL 05 San Juan de Lurigancho.

Existe una correlación lineal positiva entre la comunicación y el desempeño docente

\section{Segunda prueba de hipótesis}

\section{Hipótesis de Trabajo}

Formula del coeficiente de correlación de Pearson:
H1: Existe una correlación importante entre la motivación del equipo directivo y el desempeño docente de la I.E. 0069 Machu Picchu RED 09UGEL 05 San Juan de Lurigancho.

Ho: No Existe una correlación importante entre la motivación del equipo directivo y el desempeño docente de la I.E. 0069 Machu Picchu RED 09- UGEL 05 San Juan de Lurigancho. 


$$
R=\frac{\sum X Y-\sum X \sum Y}{\sqrt{\left(N \sum X^{2}-\left(\sum x\right)^{2}\right)\left(N \sum Y^{2}-\left(\sum Y\right)^{2}\right)}}
$$

Tabla 2. Contraste entre la dimensión motivación y desempeño docente

\begin{tabular}{|c|c|c|}
\hline & Pearson & $\begin{array}{c}\text { Desempeño } \\
\text { docente }\end{array}$ \\
\hline Motivación & $\begin{array}{c}\mathrm{R}=0.70 \\
\mathrm{P}=0.00^{*}\end{array}$ \\
\hline
\end{tabular}

$* \mathrm{P}<0.05$ significativo

Existe una correlación positiva (0.70) entre la motivación del equipo directivo y el desempeño docente de la I.E. 0069 Machu Picchu

\section{Tercera prueba de hipótesis}

\section{Hipótesis de Trabajo}

H1: Existe una correlación positiva entre liderazgo del equipo directivo y el desempeño docente de la I.E. 0069

Machu Picchu RED 09- UGEL 05 San Juan de Lurigancho.

Ho: No Existe una correlación positiva entre el liderazgo del equipo directivo y el desempeño docente de la I.E. 0069 Machu Picchu RED 09- UGEL 05 San Juan de Lurigancho

Formula del coeficiente de correlación de Pearson.

$$
R=\frac{\sum X Y-\sum X \sum Y}{\left.\sqrt{\left(N \sum X^{2}-\left(\sum x\right)^{2}\right)(} N \sum Y^{2}-\left(\sum Y\right)^{2}\right)}
$$

\begin{tabular}{|c|c|c|}
\hline & & $\begin{array}{c}\text { Desempeño } \\
\text { docente }\end{array}$ \\
\hline Liderazgo & $\begin{array}{l}\text { Pearson } \\
\text { Correlación }\end{array}$ & $\begin{array}{c}R=0.76 \\
P=0.000^{*}\end{array}$ \\
\hline
\end{tabular}

Tabla 3. Contraste entre la dimensión liderazgo y desempeño docente

${ }^{*} \mathrm{P}<0.05$ significativo $\mathrm{P}=$ probabilidad de error

Existe una correlación positiva (0.76) entre liderazgo del equipo directivo y el desempeño docente de la I.E. 0069 Machu Picchu RED 09- UGEL 05 San Juan de Lurigancho 
TABLA N 4

Descripción de las Dimensiones Eficacia del Desempeño Docente según aspectos Favorables y Desfavorables

\begin{tabular}{|l|c|c|c|c|}
\hline & \multicolumn{2}{|c|}{ Favorable } & \multicolumn{2}{c|}{ Desfavorable } \\
\hline Dimensión & n & $\%$ & n & $\%$ \\
\hline Programación curricular & 35 & 58.33 & 25 & 41.67 \\
\hline $\begin{array}{l}\text { Ejecución dirección del } \\
\text { aprendizaje }\end{array}$ & 30 & 50.00 & 30 & 50.00 \\
\hline Evaluación & 25 & 41.67 & 35 & 58.33 \\
\hline
\end{tabular}

Se observa que 58.33\% considera favorable la programación curricular; el 50\% considera favorable la ejecución de dirección del aprendizaje y el $41.67 \%$ considera favorable la evaluación

TABLA N ${ }^{\circ}$

Descripción de las dimensiones del Liderazgo del equipo Directivo según aspectos Favorables y Desfavorables

\begin{tabular}{|l|c|c|c|c|}
\hline & \multicolumn{2}{|c|}{ Favorable } & \multicolumn{2}{c|}{ Desfavorable } \\
\hline Dimensión & n & $\%$ & n & $\%$ \\
\hline Programación curricular & 50 & 83.33 & 10 & 16.67 \\
\hline $\begin{array}{l}\text { Ejecución dirección del } \\
\text { aprendizaje }\end{array}$ & 55 & 91.67 & 5 & 8.33 \\
\hline Evaluación & 57 & 95.00 & 3 & 5.00 \\
\hline
\end{tabular}

Se observa que el $83.335 \%$ considera que la motivación es favorable para el liderazgo de directores, el $91.67 \%$ considera que la motivación es favorable y el $95 \%$ considera que el liderazgo es favorable

\section{Discusión de los resultados}

El análisis correlacional de Pearson está fundamentado en la variabilidad de los datos y la ubicación exacta de la dispersión de los datos, para obtener resultados más confiables y generalizables.

En los resultados obtenidos por el coeficiente de correlación de Pearson nos presentan una alta correlación significativo entre la comunicación del equipo directivo con desempeño docente (0.739): también se encontró alta correlación significativa entre la motivación del equipo directivo y el desempeño docente de las instituciones (0.70), el estudio evidencia una alta correlación entre liderazgo del equipo directivo y el desempeño docente obteniéndose una correlación de Pearson de (0.76). Lo cual concluimos que a mayor capacidad del liderazgodirectivo mayor desempeño docente es decir el directivo y el docente deben tomar actitudes positivas, tomando una cultura donde los roles asumidos den como resultados trabajos de calidad, donde el desempeño del docente sede sin dificultades. Estos resultados concuerdan con concuerdan con Rincón Chahuillco con su 
investigación correlacional "Relación entre estilo de liderazgo del director y desempeño de docentes", De los resultados obtenidos los datos nos evidencian que existe un alto grado de correlación entre el estilo de liderazgo del director y el desempeño docente.

El presente estudio concuerda con los resultados de Flores, R. (2003) Estilos de liderazgo y su relación con el desempeño docente en el aula, encuentra la percepción y evaluación de los alumnos del quinto grado de secundaria en los colegios estatales de áreas técnicas de la USE $N^{\circ}$ 06 ATE- VITARTE. Al realizar esta investigación se llegó a la conclusión de que los docentes en su desempeño en el aula utilizan con mayor frecuencia y en forma predominante el estilo de liderazgo tolerancia a la libertad; en un nivel alto predomina el estilo de liderazgo consideración y en un nivel medio predomina el estilo de liderazgo Iniciación de estructura.

La educación presenta serias deficiencias a causa de una estructura o un sistema educativo que esta fuera del contexto a los intereses de nuestra

\section{Conclusiones}

- Los resultados parciales y generales obtenidos a través de los diferentes instrumentos de recolección de datos nos permiten afirmar que existe una correlación positiva $(0.73)$ entre la comunicación del equipo directivo y el desempeño docente de la I.E. 0069 Machu Picchu RED 09- UGEL 05 San Juan de Lurigancho

- Se concluye que existe alta correlación positiva entre la motivación del equipo directivo y el desempeño docente de la I.E. 0069 Machu Picchu RED 09- UGEL 05 San Juan de Lurigancho.

- Se establece que existe alta correlación positiva entre el liderazgo del equipo directivo y el desempeño docente de la I.E. 0069 Machu Picchu RED 09- UGEL 05 San Juan de Lurigancho.

\section{Referencia Bibliográfica}

[1] Arraluce, Delgado. (1999). "Psicología y Manejo de Empresas: Perfil del Ejecutivo Modelo". Revista Peruana de Psicología, Lima ,4 (7, nación. La vieja escuela formal y academicista sigue siendo un obstáculo al desarrollo de una verdadera propuesta educativa nacional o de una nueva escuela que genere todo un movimiento de abajo hacia arriba y de un gran esfuerzo que transforme las viejas y caducas estructuras de nuestro país. Determinamos entonces, el principio que plantea que, a mejor estilo de liderazgo implantado en la Institución Educativa, se obtendrán óptimos resultados de eficacia en el desempeño de funciones de los docentes, lo cual es beneficioso para el desarrollo personal de los maestros.

Todo cambio, innovación o desarrollo de nuevos procedimientos, involucra el temor de aquellas personas que hasta la fecha ya hicieron del trabajo un hábito de costumbre para poder cumplir con los estándares de calidad exigidos por su equipo directivo, es por ello la reacción inversa; sin embargo, todo cambio planificado tiene como meta mejorar los niveles de calidad, aminorar esfuerzos y busca la exactitud de la información solicitada.

[2] Biddle, Bruce: Contemporary research an teachers effectiveness. Citado por Barriga y Vidalón, Op. cit.

[3] Blanchard, K. \& Jonson, S. (1988) El Ejecutivo al minuto en forma. Barcelona España.

[4] Barriga Hernandez, Carlos y Vidalón, Nelly: Influencia del docente en el rendimiento del alumno. Lima, INIDE, 1975.

[5] Blanchard, K y Hersey. (1988) Liderazgo como la actividad de influenciar en la gente. Barcelona - España.

[6] Brookover, Wilbur: The Relation of social factors o teaching ability.

[7] En: Journal of Experimental Education Research. Vol. 83, 1975. pp. 195- 205.

[8] Bohn, Coylene y otros: A study of teacher's characteristics as predictors in the susccesful implementation of an innovative curriculum. USA, Informational Resources Information Center, 1971

[9] Benvenuto, Jobita, Monge, Zully \& Zanini, Marcela (2005), La Incidencia de las Relaciones Interpersonales en el Desempeño Escolar “, Venezuela.

[10] Carrera, B. (2002) Tendencias de liderazgo en el docente venezolano de la I etapa de Educación Básica. Venezuela.

[11] Chiavenato, Idalberto (1993), Administración de los Recursos Humanos, 2 edición. Ed. Mac.Graw.Hill. México. 
[12] De La Torre, Aníbal (2006), "Prácticas pedagógicas y formación de profesores. Aportes para el mejoramiento de la calidad y equidad de la educación chilena", Universidad Alberto Hurtado

[13] Gil, F (1980) Liderazgo. México: I.N

[14] Quijano, A. (2003). Características del líder. Universidad del Rosario D.C., Bogotá. Colombia.

[15] Flores, R. (2006) Estilos de liderazgo y su relación con el desempeño docente en el aula. Lima - Perú.

[16] Hernández, R., Fernández, C. \& Baptista, L. (2003). Metodología de la Investigación. Tercera edición. México: Mc Graw Hill Editores.

[17] Hemphill, JK. (1954) a proponed theory of leadership in small groups. Second preliminary report, Personnel Research Board (Ohio State University.

[18] Kasuga, L. (1999). Aprendizaje acelerado: estrategias para la potencialización del aprendizaje $\left(3^{\circ}\right.$ ed.), México: Grupo Editorial Tomo, S.A. de C.V

[19] Kotter, J.P. (1980). The general manager. New York, Free Press.

[20] Koontz, H y Weihrich, H (1998): "Administración una Perspectiva Global". Oncena Ediciòn. Mc Graw Hill, Mexico DF.7

[21] Mintzberg (1975) La Naturaleza del trabajo Directivo. Barcelona. Ed. Ariel.

[22] Musen y Conger: Desarrollo de la personalidad en el niño. México, Editorial Trillas, 1973.

[23] Muñoz y Guzmán: Una exploración de los factores determinantes del rendimiento escolar en la Educación Primaria. En: Revista del Centro de Estudios Educativo.

[24] Ortega, N (2005) El liderazgo. Revista gestiopolis. Recuperado de http://www.gestiopolis.com/el-liderazgo/
[25] Padilla, D. (2005). Percepción de los docentes, administrativos y alumnos sobre el Liderazgo y Cultura Organizacional en la Universidad Privada Antenor Orrego. Tesis inédita de Maestría, Universidad Privada Antenor Orrego, Trujillo - Perú.

[26] Paramo, D. Ramírez, E y Rodríguez, A (2005) Influencia de los estilos de dirección sobre las culturas organizacionales orientadas al mercado. Colombia: Grafiplast

[27] Quijano, A. (2003). Características del líder. Universidad del Rosario D.C., Bogotá. Colombia.

[28] Sovero, Hinostrza Franklin, Como dirigir una institución educativa. Edicion 2007.

[29] Tregoe y Zinmermann (1980). Planificacion estratégica, Gerencia, Administracion de Empresas, Gerentes.

[30] Trujillo, M. (2004), estudios de Maestría en Gestión Educativa en la Universidad Nacional Mayor de San Marcos. Lima, Perú.

[31] UNESCO (2008) Experiencias educativas de segunda oportunidad. Chile: Sol

[32] Prieto, D (1998) Comunicación educativa en el contexto latinoamericano. Revista Intervención Psicosocial $\mathrm{N}^{\circ} 7$

[33] Rincón, J. (1995) Relación entre estilo de liderazgo del director y desempeño de docentes del valle de Chumbao de la provincia de Andahuaylas. UNMSM Lima -Perú.

[34] Rosales, Mariela (2005), “Calidad sin Liderazgo. Chile: Universidad Concepción

[35] Vaillant, D (2007) Nuevas tendencias en la formación permanente del profesorado. Barceloa. Preal

[36] Valle, y Núñez 1989) La expectativa del profesor y su incidencia en el contexto institucional. Revista de educación N $^{\circ} 290$ $(293-319)$ 